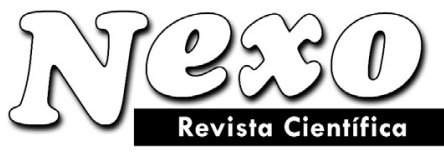

ISSN 1818-6742

Impreso en Nicaragua. www.uni.edu.ni/Nexo

Vol. 22, No. 02, pp.66-71/Diciembre 2009

\title{
El producto de convolución de la derivada de la delta de Dirac en $1-x^{2 *}$
}

\author{
M. García y M. Aguirre ${ }^{\dagger}$ \\ Núcleo Consolidado Matemática Pura y Aplicada-NUCOMPA \\ Facultad de Ciencias Exactas UNCentro, Pinto 399, \\ 7000 Tandil, Argentina. \\ e-mail: maguirre@exa.unicen.edu.ar
}

(recibido/received: 10-Marzo-2009; aceptado/accepted: 20-Noviembre-2009)

\section{RESUMEN}

En este artículo se le dio un sentido al producto de convolución de $\delta^{(k-1)}\left(1-x^{2}\right) * \delta^{(\ell-1)}\left(1-x^{2}\right)$.

Como caso particular se obtuvo una fórmula del producto de convolución de $\delta\left(1-x^{2}\right) * \delta\left(1-x^{2}\right)$ (C.f. fórmula (38)).

Palabras Claves: Convolución; Producto

ABSTRACT

In this paper we give a sense to distribution convolution product of $\delta^{(k-1)}\left(1-x^{2}\right) * \delta^{(\ell-1)}\left(1-x^{2}\right)$.

As a particular case we obtain a formula to convolution product of $\delta\left(1-x^{2}\right) * \delta\left(1-x^{2}\right)$ (C.f. formula (38)).

Keywords: Convolution; Product.

\footnotetext{
${ }^{*}$ Este trabajo fue parcialmente soportado por la Comisión de Investigación Científica de la Provincia de Buenos Aires (C.I.C.), Argentina.

${ }^{\dagger}$ Autor para la correspondencia
} 


\section{INTRODUCCIÓN}

Sea $x$ un punto en $R$ y $\lambda$ un complejo en $C$, donde con $R$ se designa a los números reales y con $C$ a los números complejos.

Consideremos la función $\left(1-x^{2}\right)_{+}^{\lambda}$ igual $\left(1-x^{2}\right)_{+}^{\lambda}$ para $1-x^{2}>0$, y cero para $1-x^{2} \leq 0$. La función generalizada correspondiente es definida de la siguiente forma,

$$
\left\langle\left(1-x^{2}\right)_{+}^{\lambda}, \varphi\right\rangle=\int_{-\infty}^{+\infty}\left(1-x^{2}\right)_{+}^{\lambda} \varphi(x) d x=\int_{-1}^{+1}\left(1-x^{2}\right)^{\lambda} \varphi(x) d x
$$

Para toda $\varphi$ en $K$, donde $K$ es el conjunto de funciones con derivadas continuas de todos los órdenes y con soporte acotado ([1], página 195).

La integral (1) converge para $\operatorname{Re} \lambda>1$, y para otros valores de $\lambda$ puede ser regularizada (analíticamente prolongada) de acuerdo con la expresión dada en ([2], capítulo I, sección 3).

De (([2]), página 183), $\left(1-x^{2}\right)_{+}^{\lambda}$ es una función generalizada la cual es analítica en todo punto excepto en los puntos $\lambda=-k$, donde $k$ es cualquier entero positivo en cuyos puntos tiene polos simples con residuo

$$
\underset{\lambda=-k, k=1,2, \ldots}{\operatorname{Re} s\left(1-x^{2}\right)_{+}^{\lambda}}=\lim _{\lambda \rightarrow-k}(\lambda+k)\left(1-x^{2}\right)_{+}^{\lambda}=\frac{(-1)^{k-1}}{(k-1) !} \delta^{(k-1)}\left(1-x^{2}\right) .
$$

Se calculará la transformada de Fourier de $\left(1-x^{2}\right)_{+}^{\lambda}$. Primeramente se restringen las consideraciones a valores de $\lambda$ tales que $-1<\operatorname{Re} \lambda<0$.

Se considera la expresión:

$$
\begin{aligned}
F\left\{e^{-x \tau}\left(1-x^{2}\right)_{+}^{\lambda}\right\} & =\int_{-\infty}^{+\infty} e^{-x \tau} e^{\sigma x i}\left(1-x^{2}\right)_{+}^{\lambda} d x= \\
& =\int_{-1}^{+1}\left(1-x^{2}\right)^{\lambda} e^{i x s} d x
\end{aligned}
$$

donde

$$
s=\sigma+i \tau
$$

Ahora usando la fórmula ([2], fórmula 2, página 185),

$$
\int_{-1}^{+1}\left(1-x^{2}\right)^{v-1} e^{i \mu x} d x=\sqrt{\pi}\left(\frac{2}{\mu}\right)^{v-1 / 2} \Gamma(v) J_{v-1 / 2}(\mu), \quad \operatorname{Re}(v)>0
$$

se tiene

donde

$$
F\left\{e^{-x \tau}\left(1-x^{2}\right)_{+}^{\lambda}\right\}=\sqrt{\pi} 2^{\lambda+1 / 2} s^{-\lambda-1 / 2} \Gamma(\lambda+1) J_{\lambda+1 / 2}(s)
$$

$$
J_{\alpha}(z)=\left(\frac{z}{2}\right)^{\alpha} \sum_{j \geq 0} \frac{(-1)^{j}(z / 2)^{2 j}}{j ! \Gamma(\alpha+j+1)}
$$

y $s$ es definida por (4). 
La fórmula (6), usando (7) puede ser escrita en la siguiente forma

$$
F\left\{e^{-x \tau}\left(1-x^{2}\right)_{+}^{\lambda}\right\}=\sqrt{\pi} \Gamma(\lambda+1) \sum_{j \geq 0} \frac{(-1)^{j}(s / 2)^{2 j}}{j ! \Gamma(\lambda+j+3 / 2)}
$$

Como $\tau \rightarrow 0^{+}$se concluye que $e^{-x \tau}\left(1-x^{2}\right)_{+}^{\lambda}$ converge a $\left(1-x^{2}\right)_{+}^{\lambda}$ en el sentido de las funciones generalizadas, luego su transformada de Fourier converge a la transformada de Fourier de $\left(1-x^{2}\right)_{+}^{\lambda}$.

De (8) y tomando límite cuando $\tau \rightarrow 0^{+}$, se llega a la siguiente fórmula

$$
F\left\{\left(1-x^{2}\right)_{+}^{\lambda}\right\}=\sqrt{\pi} \Gamma(\lambda+1) \sum_{j \geq 0} \frac{(-1)^{j}}{2^{2 j} j ! \Gamma(\lambda+j+3 / 2)}(\sigma+i 0)^{2 j}
$$

donde

$$
(\sigma+i 0)^{\lambda}=\lim _{\tau \rightarrow 0^{+}}(\sigma+i \tau)^{\lambda} \quad([2], \text { página } 59)
$$

Ahora por prolongación analítica ([2], página 171) esta fórmula se extendería para todos los valores de $\lambda$ tales que $\lambda \neq-1,-2,-3, .$.

Dividiendo ambos términos de la ecuación por $\Gamma(\lambda+1)$, se obtiene una función entera de $\lambda$ en ambos lados de la ecuación, así que para todo $1 \lambda$ se puede escribir

$$
F\left\{\frac{\left(1-x^{2}\right)_{+}^{\lambda}}{\Gamma(\lambda+1)}\right\}=\sqrt{\pi} \sum_{j \geq 0} \frac{(-1)^{j}}{2^{2 j} j ! \Gamma(\lambda+j+3 / 2)}(\sigma+i 0)^{2 j}
$$

donde

$$
F\{f(x)\}=\int_{-\infty}^{+\infty} f(x) e^{i \sigma x} d x
$$

y

$$
f(x)=\frac{1}{2 \pi} \int_{-\infty}^{+\infty} F\{f(x)\}(y) e^{-i \sigma y} d y
$$

Por otra parte, de (11) usando (2) y considerando las fórmulas

si $\lambda$ es un entero no negativo ([2], página 60) y

$$
(\sigma+i 0)^{\lambda}=\sigma^{\lambda}
$$

$$
\underset{z=-\ell, \ell=0,1,2, \ldots}{\operatorname{Re}} \Gamma(z)=\frac{(-1)^{\ell}}{\ell !}
$$

donde $\Gamma(z)$ es la función gamma definida por:

$$
\Gamma(z)=\int_{0}^{+\infty} e^{-x} x^{z-1} d x
$$

Se obtiene la transformada de Fourier de $\delta^{(k-1)}\left(1-x^{2}\right)$, 


$$
F\left\{\delta^{(k-1)}\left(1-x^{2}\right)\right\}=\sqrt{\pi} \sum_{j \geq 0} \frac{(-1)^{j}}{2^{2 j} j ! \Gamma(j+3 / 2-k)}(\sigma)^{2 j}
$$

para $k=1,2,3, \ldots$

De (17) y usando la fórmula

$$
F\left\{\delta^{(2 m)}(x)\right\}=(-1)^{m} \sigma^{2 m} \quad([2], \text { página 60) }
$$

se tiene

$$
F\left\{\delta^{(k-1)}\left(1-x^{2}\right)\right\}=\sqrt{2 \pi} \sum_{j \geq 0} \frac{1}{2^{2 j} j ! \Gamma(j+3 / 2-k)} F\left\{\delta^{(2 j)}(x)\right\}
$$

De (19) se obtiene un desarrollo tipo Taylor de $\delta^{(k-1)}\left(1-x^{2}\right)$,

$$
\delta^{(k-1)}\left(1-x^{2}\right)=\frac{1}{2 \sqrt{\pi}} \sum_{j \geq 0} \frac{1}{2^{2 j} j ! \Gamma(j+3 / 2-k)} \delta^{(2 j)}(x)
$$

\section{EL PRODUCTO DE CONVOLUCIÓN DE $\delta^{(k-1)}\left(1-x^{2}\right) * \delta^{(\ell-1)}\left(1-x^{2}\right)$}

La distribución $\delta^{(2 m)}(x)$ es de clase $O_{C}^{\prime}$, donde $O_{C}^{\prime}$ es el dual del espacio $O_{C}$, y $O_{C}$ son funciones que decrecen rápidamente, por tanto $\delta^{(2 m)}(x) \in S^{\prime}$. Considerando el teorema clásico de Laurent Schwartz ([S], página 268, fórmula (VII, 8,5))

$$
\mathbf{F}\left\{\delta^{(2 m)}(x) * \delta^{(2 \ell)}(x)\right\}=(2 \pi) \mathbf{F}\left\{\delta^{(2 m)}(x)\right\} . \mathbf{F}\left\{\delta^{(2 \ell)}(x)\right\}
$$

y la fórmula (18) se tiene,

$$
\begin{aligned}
\mathbf{F}\left\{\delta^{(2 m)}(x) * \delta^{(2 \ell)}(x)\right\} & =2 \pi(-1)^{m+\ell} \sigma^{2(m+\ell)} \\
& =2 \pi \mathbf{F}\left\{\delta^{(2(m+\ell))}(x)\right\}
\end{aligned}
$$

De la fórmula (22), se obtiene la fórmula

$$
\delta^{(2 m)}(x) * \delta^{(2 \ell)}(x)=\delta^{(2(m+\ell))}(x)
$$

Ahora de (20) y considerando que $\delta^{(2 m)}(x)$ está en $O_{C}^{\prime}$ entonces $\delta^{(k-1)}\left(1-x^{2}\right)$ es de clase $O_{C}^{\prime}$, y el producto de convolución $\delta^{(k-1)}\left(1-x^{2}\right) * \delta^{(\ell-1)}\left(1-x^{2}\right)$ existe.

Por tanto, de (20) y considerando (24) se tiene

$$
\begin{gathered}
\delta^{(k-1)}\left(1-x^{2}\right) * \delta^{(\ell-1)}\left(1-x^{2}\right)= \\
=\frac{\sqrt{\pi}}{2 \pi} \sum_{j \geq 0} \frac{(-1)^{j}}{j ! 2^{2 j} \Gamma\left(j+\frac{3}{2}-k\right)} \frac{\sqrt{\pi}}{2 \pi} \sum_{r \geq 0} \frac{(-1)^{r}}{r ! 2^{2 r} \Gamma\left(r+\frac{3}{2}-\ell\right)}\left[\delta^{(2 j+2 r)}(x)\right]
\end{gathered}
$$

ahora, tomando en cuenta la fórmula 


$$
\left(\begin{array}{l}
\alpha \\
k
\end{array}\right)=\frac{\Gamma(\alpha+1)}{k ! \Gamma(\alpha-k+1)}
$$

y la propiedad

$$
\sum_{j=0}^{n}\left(\begin{array}{c}
n-\ell+\frac{1}{2} \\
j
\end{array}\right)\left(\begin{array}{c}
n-k+\frac{1}{2} \\
n-j
\end{array}\right)=\left(\begin{array}{c}
2 n-\ell-k+1 \\
n
\end{array}\right)
$$

se tiene,

$$
\begin{gathered}
\sum_{j \geq 0} \sum_{r \geq 0} \frac{1}{2^{2(j+r)} j ! r ! \Gamma\left(j+\frac{3}{2}-k\right) \Gamma\left(r+\frac{3}{2}-\ell\right)}= \\
\sum_{n \geq 0} \sum_{j=0}^{n} \frac{1}{2^{2(j+r)} j !(n-j) !} \frac{1}{\Gamma\left(j+\frac{3}{2}-k\right) \Gamma\left(n-j+\frac{3}{2}-\ell\right)}
\end{gathered}
$$

donde $j+r=n$, por tanto,

$$
\begin{gathered}
\frac{1}{\Gamma\left(j+\frac{3}{2}-k\right) \Gamma\left(n-j+\frac{3}{2}-\ell\right)}= \\
=\sum_{n \geq 0}\left\{\sum_{j=0}^{n}\left(\begin{array}{c}
n-\ell+\frac{1}{2} \\
j
\end{array}\right) \frac{1}{\Gamma\left(n-\ell+\frac{3}{2}\right)}\left(\begin{array}{c}
n-k+\frac{1}{2} \\
n-j
\end{array}\right) \frac{1}{\Gamma\left(n-k+\frac{3}{2}\right)}\right\}= \\
=\sum_{n \geq 0} \frac{1}{\Gamma\left(n-\ell+\frac{3}{2}\right) \Gamma\left(n-k+\frac{3}{2}\right)}\left(\begin{array}{c}
2 n-k-\ell+1 \\
n
\end{array}\right) .
\end{gathered}
$$

entonces,

$$
\begin{aligned}
& \sum_{j \geq 0} \sum_{r \geq 0} \frac{1}{2^{2(j+r)} j ! r ! \Gamma\left(j+\frac{3}{2}-k\right) \Gamma\left(r+\frac{3}{2}-\ell\right)}= \\
& \sum_{n \geq 0} \frac{1}{2^{2 n} n ! \Gamma\left(n-\ell+\frac{3}{2}\right) \Gamma\left(n-k+\frac{3}{2}\right)} \frac{\Gamma(2 n-k-\ell+2)}{\Gamma(n-k-\ell+2)} .
\end{aligned}
$$

De (25) y usando (30) se obtiene

$$
\begin{gathered}
\delta^{(k-1)}\left(1-x^{2}\right) * \delta^{(\ell-1)}\left(1-x^{2}\right)= \\
=\frac{1}{4 \pi} \sum_{n \geq 0} A_{n, k, \ell} \delta^{(2 n)}(x)
\end{gathered}
$$

donde,

$$
A_{n, k, \ell}=\frac{\Gamma(2 n-k-\ell+2)}{2^{2 n} n ! \Gamma\left(n-\ell+\frac{3}{2}\right) \Gamma\left(n-k+\frac{3}{2}\right) \Gamma(n-k-\ell+2)} .
$$

En particular, haciendo $k=\ell=1$ en (31), (32) y usando la fórmula de duplicación de Legendre

$$
\Gamma(2 z)=2^{2 z-1} \pi^{-\frac{1}{2}} \Gamma(z) \Gamma\left(z+\frac{1}{2}\right)
$$

se tiene 


$$
\begin{gathered}
\delta\left(1-x^{2}\right) * \delta\left(1-x^{2}\right)=\frac{1}{4 \pi} \sum_{n \geq 0} A_{n, 1,1} \delta^{(2 n)}(x) \\
=\frac{1}{4 \pi} \sum_{n \geq 0} \frac{\Gamma(2 n)}{2^{2 n} n ! \Gamma\left(n+\frac{1}{2}\right) \Gamma\left(n+\frac{1}{2}\right) \Gamma(n)} \delta^{(2 n)}(x) \\
=\frac{1}{4 \pi(\sqrt{\pi})} \sum_{n \geq 0} \frac{1}{n ! \Gamma\left(n+\frac{1}{2}\right)} \delta^{(2 n)}(x) .
\end{gathered}
$$

Por otra parte, considerando la función definida por:

$$
\left(1-\frac{x^{2}}{4}\right)_{+}^{\lambda}=\left\{\begin{array}{ccc}
\left(1-\frac{x^{2}}{4}\right)^{\lambda} & \text { si } & 1-\frac{x^{2}}{4}>0 \\
0 & \text { si } & 1-\frac{x^{2}}{4} \leq 0
\end{array}\right.
$$

y considerando las fórmulas (3), (6), (7), (8), (11), (17), (18) y (20) se obtiene la siguiente fórmula,

$$
\delta^{(k-1)}\left(1-\frac{x^{2}}{4}\right)=\frac{2 \sqrt{\pi}}{2 \pi} \sum_{j \geq 0} \frac{1}{j ! \Gamma\left(j+\frac{3}{2}-k\right)} \delta^{(2 j)}(x) .
$$

De (33) y (35) se obtiene la siguiente fórmula,

De (36) se concluye que

$$
\begin{gathered}
\delta\left(1-x^{2}\right) * \delta\left(1-x^{2}\right)= \\
=\frac{1}{4 \pi \sqrt{\pi}} \sum_{n \geq 0} \frac{\delta^{(2 n)}(x)}{n ! \Gamma\left(n+\frac{1}{2}\right)} \\
=\frac{1}{4 \pi} \delta\left(1-\frac{x^{2}}{4}\right) .
\end{gathered}
$$

o equivalentemente

$$
\delta\left(1-x^{2}\right) * \delta\left(1-x^{2}\right)=\frac{1}{4 \pi} \delta\left(1-\frac{x^{2}}{4}\right)
$$

$$
\delta\left(1-x^{2}\right) * \delta\left(1-x^{2}\right)=\frac{1}{4 \pi} \delta\left(1-\left(\frac{x}{2}\right)^{2}\right)
$$

\section{REFERENCIAS}

[1] Zemanian, A. H. 1965. Distribution theory and Transform Analysis. Mc Graw Hill Book Company. New York.

[2] Gel'fand and Shilov. 1964. Generalized Functions- Vol. I-Academic Press, New York

[3] Schwartz Laurent. 1973. Theorie des Distributions.Hermann, París.

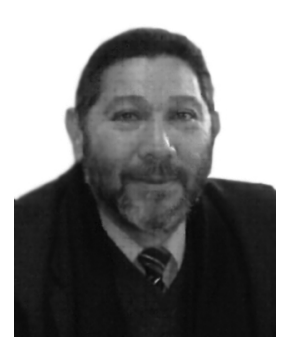

Manuel A. Aguirre, es Profesor y Decano de la Facultad de Ciencias Exactas de la Universidad Nacional del Centro de la Provincia de Buenos Aires, Núcleo Consolidado Matemática Pura y Aplicada-NUCOMPA Facultad de Ciencias Exactas UNCentro

Paraje Arroyo Seco, 7000-Tandil

Provincia de Buenos Aires, Argentina

Tel.: +54 2293439657

E-mail: maguirre@exa.unicen.edu.ar 